\title{
FULLY NUCLEAR OPERATORS
}

\author{
BY C. P. STEGALL AND J. R. RETHERFORD ${ }^{1}$ \\ Communicated by M. H. Protter, March 11, 1970
}

1. Introduction. This note is an outgrowth of a study of the following conjecture of Grothendieck [5, Chapter II, p. 47].

(C) Let $E$ and $F$ be Banach spaces such that every $T \in \mathcal{L}(E, F)$, the continuous linear operators from $E$ to $F$, is nuclear. Then either $E$ or $F$ is finite dimensional.

Recall that an operator $T: E \rightarrow F$ is nuclear if there exists $\left(f_{i}\right) \subset E^{\prime}$, $\left(x_{i}\right) \subset F$ such that

$$
T x=\sum_{i=1}^{\infty} f_{i}(x) x_{i} \quad \text { for every } x \in E \text { and } \quad \sum_{i=1}^{\infty}\left\|f_{i}\right\|\left\|x_{i}\right\|<+\infty .
$$

Every nuclear operator obviously has the property that the image of an unconditionally convergent series is absolutely convergent. An operator with this latter property is called an absolutely summing operator [14] (Grothendieck called these operators "semi-intégrale à droit"). It can happen in nontrivial cases, e.g. $\mathcal{L}\left(l_{1}, l_{2}\right)$, that all continuous linear operators are absolutely summing [5], [9]. Thus, one approach to (C) is to seek a criterion guaranteeing the existence of nonabsolutely summing operators between Banach spaces. Such a criterion is developed in [22].

To our knowledge (C) has been considered only in [2] and the important recent paper of Lindenstrauss and Pełczyński [9]. Although we are unable to solve (C) in the generality asserted by Grothendieck, we are able to give an affirmative answer to (C) under a somewhat more restricted hypothesis. However, we should mention that if $(\mathrm{C})$ is true as asserted by Grothendieck then our hypotheses must be satisfied.

We need two results from [22].

Theorem A. Let $E$ and $F$ be infinite dimensional Banach spaces. Then there is an infinite dimensional subspace $E_{0}$ of $E$ and an operator $T: E_{0} \rightarrow F$ such that $T$ is not absolutely summing.

AMS 1970 subject classifications. Primary 46A99, 47B10.

Key words and phrases. Absolutely summing operator, nuclear operator, fully nuclear operator, and completely nuclear operator.

1 Research supported by NSF-GP 11761. 
Theorem B. Let $E$ and $F$ be infinite dimensional Banach spaces. Then there is an infinite dimensional subspace $F_{0}$ of $F$ and an operator $T: E \rightarrow F_{0}$ such that $T^{\prime}$ is not absolutely summing.

Proofs of these theorems and related results will appear elsewhere.

2. Fully nuclear operators. Many of the difficulties in the theory of nuclear operators arise because the representation of a nuclear operator $T$ from a space $E$ to a space $F$ depends on the range $F$ and not the image $T(E)$. To avoid these difficulties we make the following definition.

Definition 1. A bounded operator $T: E \rightarrow F, E$ and $F$ Banach spaces, is fully nuclear if the astriction $T_{a}: E \rightarrow T(E)$ is nuclear. We will denote by $N(E, F)$ and $F N(E, F)$ the nuclear and fully nuclear operators from $E$ to $F$ respectively.

Grothendieck [2, p. 40] has given a general method for obtaining nuclear mappings which are not fully nuclear. We will see below that, in a certain sense, each nuclear operator can be obtained from a fully nuclear operator.

Before proceeding to the results we should mention that it is immaterial whether we consider the astriction to the image or the closure of the image in Definition 1.

We now state our main result:

Theorem 1. Let $E$ and $F$ be Banach spaces and suppose $\mathcal{L}(E, F)$ $=F N(E, F)$. Then $E$ or $F$ is finite dimensional.

The converse is also true.

The proof of Theorem 1 is immediate from Theorem B. Of course, Theorem 1 is the fully nuclear analog of (C).

Using the results of $[22]$ we can prove

THEOREM 2. If $E$ and $F$ are infinite dimensional Banach space with $F$ isomorphic to a conjugate space then there is a quotient space $G$ of $F$ and an operator $T: E \rightarrow G$ such that $T$ is not nuclear.

3. The linear and "ideal" structure of $F N(E, F)$. It is well known [7], [13] that $N(E, F)$ is a linear space and that if $S \in \mathcal{L}(E, F)$, $T \in N(F, G), R \in \mathcal{L}(G, H)$ then

$$
T \circ S \in N(E, G) \text { and } R \circ T \in N(F, H)
$$

for all Banach spaces $E, F, G$, and $H$.

Unfortunately $F N(E, F)$ is not so well behaved.

Theorem 3. Let $T \in N(E, F)$ and suppose $\operatorname{Ker} T=T^{-1}(0)$ has infinite dimension. Then $T$ has a representation $T=T_{1}-T_{2}$ where $T_{1}$, $T_{2} \in F N(E, F)$. 
From Theorem 3 and the construction of Grothendieck [2, p. 40] it follows that $F N(E, F)$ is not, in general, a linear space. There are important cases where $F N(E, F)$ is a linear space.

The ideal structure of $F N(E, F)$ is also pathological. It is clear that $S T$ is fully nuclear whenever $T$ is fully nuclear. If $R: E \rightarrow F$ is such that the closure of $R(E)$ is $F$ and $T \in F N(F, G)$ than $T R$ is fully nuclear. However, in general, $T R$ need not be fully nuclear whenever $T$ is. This fact is a consequence of

THEOREM 4. Every nuclear operator is the restriction of a fully nuclear operator.

Theorem 4 and our next result together show that every nuclear operator is "between" two fully nuclear operators.

THEOREM 5. Let $T: E \rightarrow F$ be nuclear and suppose that the dimension of $T(E)$ is infinite. Then there exists an infinite dimensional subspace $E_{0}$ of $E$ such that the restriction of $T$ to $E_{0}$ is fully nuclear.

4. A characterization of Hilbert spaces. In this section we give an operator characterization of Hilbert spaces.

Theorem 6. Let $T \in N(E, F)$ and suppose $\overline{T(E)}$ is complemented in F. Then $T \in F N(E, F)$.

As an easy corollary we obtain

THEOREM 7. If $F$ is isomorphic to a Hilbert space (in particular if $F$ is finite dimensional) then for any Banach space $E, N(E, F)=F N(E, F)$.

Since the mappings of finite rank are dense in $N(E, F)$ with the nuclear norm [13], we obtain

THEOREM 8. For all Banach space $E, F, F N(E, F)$ is dense in $N(E, F)$ (with the nuclear norm).

As a partial converse to Theorem 6 we have

THEOREM 9. Let $F$ be a reflexive space such that

(i) each subspace of $F$ has the approximation property and

(ii) $N(E, F)=F N(E, F)$ for each Banach space $E$. Then every closed subspace of $F$ is complemented.

THEOREM 10. Suppose $F$ is a Banach space satisfying the conditions of Theorem 9. Suppose, moreover, that the canonical map from $F N(E, F)$ into $N(E, F)$ is an isometry for each Banach space $E$. Then $F$ is isometrically isomorphic to a Hilbert space. Moreover, any Hilbert space $F$ satisfies all the above conditions. 
We should mention that (i) of Theorem 9 is probably superfluous. Indeed, Grothendieck $[2, \S 5$, no. 2$]$ has conjectured that every reflexive Banach space has the approximation property. Also, Theorem 9 probably characterizes the isomorphs of Hilbert space. It is an unsolved problem as to whether the conclusion of Thecrem 9 characterizes the isomorphs of Hilbert space.

5. An analog of a problem of Grothendieck. It is very easy to prove that if $T \in N(E, F)$ then $T^{\prime} \in N\left(F^{\prime}, E^{\prime}\right)$ [2], [13]. The converse problem is still unsolved and apparently difficult. (See [2] to see how this problem relates to the approximation problem.) The analogous problem for fully nuclear operators is solved in the following theorem.

THEOREM 11. There is a nonfully nuclear operator $T$ whose adjoint $T^{\prime}$ is fully nuclear.

6. Completely nuclear operators. We give the definition dual to Definition 1.

Definition 2. A bounded operator $T: E \rightarrow F$ is completely nuclear if $T_{K}$, defined by

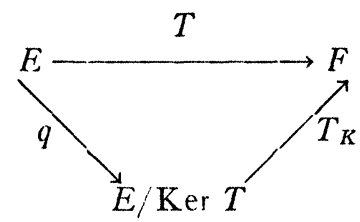

where $q$ is the quotient map, is nuclear.

The standard theorems between operators and their adjoints show the relationship between fully nuclear and completely nuclear operators. There are results dual to those of Theorems 1-11 for completely nuclear operators. We will not go into details here. We will mention only the following results (see [21]).

Theorem 12. A Banach space $E$ is an $\mathfrak{L}_{\infty}$-space ${ }^{2}$ if and only if $N(E, F)=F N(E, F)$ for every Banach space $F$.

Let $C N(E, F)$ denote the completely nuclear operators from $E$ to $F$.

THEOREM 13. A Banach space $E$ is an $\mathfrak{L}_{1}$-space ${ }^{2}$ if and only if $N(F, E)=C N(F, E)$ for all Banach spaces $F$.

Let $A S(E, F)$ denote the absolutely summing operators from $E$ to $F$. From Theorem 12 and [9] we obtain

Theorem 14. Let $E$ have an unconditional Schauder basis. Then

\footnotetext{
${ }^{2}$ The spaces of Theorems 12 and 13 are those of [9], [10].
} 
$A S(E, F)=N(E, F)$ for all Banach spaces $F$ if and only if $E$ is isomorphic to $c_{0}$.

\section{BIBLIOGRAPHY}

1. S. Banach, Théorie des opérations linéaires, Monografie Mat., PWN, Warsaw, 1932; reprint, Chelsea, New York, 1955. MR 17, 175.

2. A. Grothendieck, Produits tensoriels topologiques et espaces nucléaires, Mem. Amer. Math. Soc. No. 16(1955). MR 17, 763.

3. - - Résumé de la théorie métrique des produits tensoriels topologiques, Bol. Soc. Mat. São Paulo 8 (1953), 1-79. MR 20 \# 1194.

4. - - Sur certaines classes de suites dans les espaces de Banach et le théorème de Dvoretzky-Rogers, Bol. Soc. Mat. São Paulo 8 (1953), 81-110. MR 20 \# 1195.

5. S. Kakutani, Some characterizations of Euclidean space, Jap. J. Math. 16 (1939), 93-97. MR 1, 146.

6. J. Lindenstrauss, Extension of compact operators, Mem. Amer. Math. Soc. No. 48 (1964). MR 31 \#3828.

7. - On a certain subspace of $l_{1}$, Bull. Acad. Polon. Sci. Sér. Sci. Math. Astronom. Phys. 12 (1964), 539-542. MR 30 \#5153.

8. - On the modulus of smoothness and divergent series in Banach spaces, Michigan Math. J. 10 (1963), 241-252. MR 29 \#6316.

9. J. Lindenstrauss and A. Pełczyński, Absolutely summing operators in $\mathfrak{L}_{p}$-spaces and their applications, Studia Math. 29 (1968), 275-326. MR 37 \#6743.

10. J. Lindenstrauss and H. Rosenthal, The $\mathfrak{L}_{p}$-spaces, Israel J. Math. 7 (1969), 325-349.

11. A. Pełczyński, Projections in certain Banach spaces, Studia Math. 19 (1960), 209-228. MR 23 \#A3441.

12. M. I. Kadec and A. Pełczyński, Bases, lacunary sequences and complemented subspaces in the spaces $L_{p}$, Studia Math. 21 (1961/62), 161-176. MR 27 \#2851.

13. A. Pietsch, Nukleare lokalkonvex Raüme, Akademie-Verlag, Berlin, 1965. MR 31 \#6114.

14. - Absolut p-summierende Abbildungen in normierten Raümen, Studia Math. 28 (1966/67), 333-353. MR 35 \#7162.

15. - Quasinukleare Abbildungen in normierten Raümen, Math. Ann. 165 (1966), 76-90. MR 33 \#6412.

16. H. Rosenthal, Projections onto translation-invariant subspaces of $L^{p}(G), \mathrm{Mem}$. Amer. Math. Soc. No. 63 (1966). MR 35 \#2080.

17. - On complemented and quasi-complemented subspaces of quotients of $C(S)$ for Stonian S., Proc. Nat. Acad. Sci. U. S. A. 60 (1968), 1165-1169. MR 37 \#6740.

18. L. Schwartz, Séminaire Schwartz de al Faculté des Sciences de Paris 1953/54, Produits tensoriels topologiques d'espaces vectorieles topologiques. Espaces vectoriels tolopoquiques nucléaires. Applications, Secrétariat mathématique, Paris, 1954. MR 17,764 .

19. A. Sobczyk, Projections in Minkowski and Banach spaces, Duke Math. J. 8 (1941), 78-106. MR 2, 220.

20. - Projection of the space $(m)$ on its subspace $\left(c_{0}\right)$, Bull. Amer. Math. Soc. 47 (1941), 938-947. MR 3, 205.

21. C. P. Stegall, An operator characterization of $\mathfrak{L}_{1}$ and $\mathfrak{L}_{\infty}$-spaces, Bull. Polon. Acad. Sci. (to appear).

22. C. P. Stegall and J. R. Retherford, Fully nuclear and completely nuclear operators with applications to $\mathfrak{L}_{1}$ and $\mathfrak{L}_{\infty}$-spaces. (to appear).

Louisiana State University, Baton Rouge, Louisana 70803 